\title{
Performance and Scalability of Self-Organizing Hierarchical Ad Hoc Wireless Networks
}

\author{
Suli Zhao, Ivan Seskar and Dipankar Raychaudhuri \\ WINLAB, Rutgers University \\ 73 Brett Road, Piscataway, NJ 08854 \\ \{sulizhao, seskar, ray\}@winlab.rutgers.edu
}

\begin{abstract}
A novel self-organizing hierarchical architecture is proposed for improving the performance and scalability properties of ad hoc wireless networks. This paper presents the results of a simulation study of performance and throughput capacity of a specific three-tier hierarchical ad hoc network with 802.11 radios, forwarding nodes and access points. The performance of the proposed hierarchical network is evaluated for two well-known classes of ad hoc routing protocols: Dynamic Source Routing (DSR) and Ad hoc On-demand Distance Vector (AODV), and compared with that of a conventional "flat" ad hoc network. The results for an example sensor network scenario show significant capacity increases with the hierarchical architecture for both DSR and AODV cases. Modifications to ad hoc routing metrics for energy efficiency are also considered. The scalability properties of the three-tier hierarchy are studied further in terms of the achievable system capacity as a function of the relative densities of sensor nodes, forwarding nodes and access points. It is shown that the capacity of the three-tier hierarchical network scales well when the number of forwarding nodes and access points are increased in the right proportions.
\end{abstract}

Index Terms-Hierarchical ad hoc networks, performance evaluation, routing.

\section{INTRODUCTION}

Ad hoc networks in which radio nodes communicate via multi-hop routing have long been considered for tactical military communications without wired infrastructure. More recently, ad hoc radio techniques have migrated to dual-use and commercial scenarios such as sensor networks, home computing and public wireless LAN. While ad hoc wireless networks offer important rapid deployment and cost benefits, the traditional "flat" multi-hop routing approach does not scale well, i.e. throughput per node decreases and delay increases as the number of nodes in the system becomes large. In [1], Gupta and Kumar obtain the throughput capacity of ad hoc wireless networks, which decreases as $\Theta(1 / \sqrt{n})$ per node, as the number of nodes $(n)$ increases. This motivates consideration of more scalable ad hoc network architecures, possibly based on hierarchical approaches. In addition, potential ad hoc network applications (such as sensor arrays) involve traffic flows to and from the Internet in addition to peer-topeer communication between nodes, thus requiring effective hierarchical integration with the wired infrastructure.

Based on the above considerations, we are investigating a new class of self-organizing hierarchical ad hoc wireless networks with improved scaling properties and more natural

Research supported by NJ Commission of Science and Technology grant \#03-2042-007-12. integration with the wired Internet. The network is designed to provide hierarchical scaling of throughput with bounded delay, while retaining some of the flexibility and cost advantage of an infrastructure-less ad hoc network. Major design considerations for the proposed hierarchical ad hoc network include a discovery and topology establishment protocol for self-organization, a MAC protocol for efficient use of radio resources, and a routing protocol to support multi-hop packet transport.

Many routing protocols have been proposed for ad hoc networks, among which DSR [2] and AODV [3] are two of the most popular. Most of the routing protocols are designed for "flat" architecture, but they may also be used in hierarchical scenarios with appropriate modifications. Our objective is to evaluate how different routing protocols work in the hierarchical mode, and measure the resulting system capacity and user performance. We apply DSR and AODV routing to a specific hierarchical network with three tiers of 802.11 radio nodes defined as "sensors", "forwarding nodes" and "access points" and (using an $n s-2$ simulation model) evaluate the resulting system performance. Optimization of DSR routing metrics in terms of energy efficiency is also considered briefly.

After evaluating the comparative performance of the proposed three-tier hierarchical network with different ad hoc routing protocols, we focus on its scaling properties. Specifically, the goal is to determine the appropriate ratios of access points and forwarding nodes as a function of offered traffic load from low-tier (sensor) nodes, for the system capacity to scale linearly. This capacity scaling problem is investigated further with simulations of hierarchical network capacity with different forwarding node and access point densities. The results provide an understanding of the appropriate ratio of nodes at each level of the hierarchy required to design a wellbalanced and scalable network.

The rest of this paper is organized as follows. In the next section we introduce the proposed hierarchical ad hoc network. In Section III we discuss the routing protocols and alternative energy-aware metric, and the modifications made to DSR and AODV so that they can be applied to the hierarchical mode. The simulation model and the performance results with both the hierarchical and flat architectures for an example "sensor network" scenario are presented in Section IV and V. Section VI gives a regular planar network model for studying the hierarchical capacity scalability and the related simulation results. The last section summarizes the main results and 
outlines our future work.

\section{THREE-TIER HIERARCHICAL AD HOC NETWORK}

The proposed network architecture is based on three tiers of wireless devices: low-power "sensor nodes" with limited functionality, higher-power "radio forwarding nodes" that route packets between radio links, and "access points" that route packets between radio links and the wired infrastructure. Their functions are summarized below (see Fig. 1).

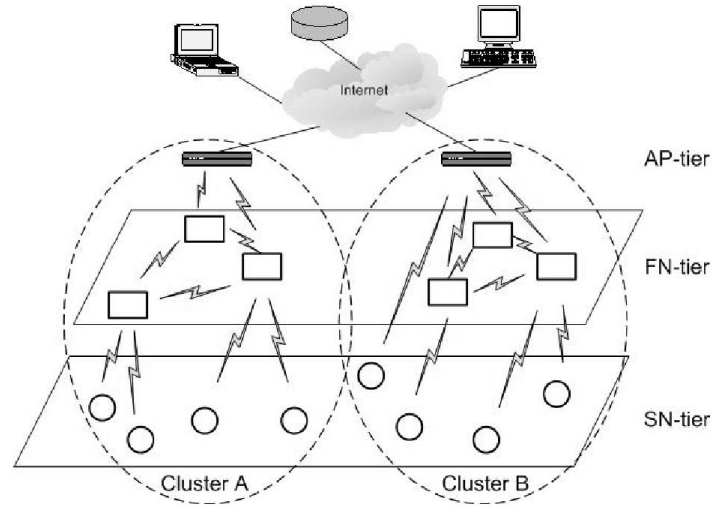

Fig. 1. Hierarchical ad hoc network architecture

- Sensor Node (SN): The sensor node is in the lowest tier and (unlike the traditional flat network model) does not offer multi-hop routing capability to its neighbors. SN's route packets via higher tier nodes, which may be either forwarding nodes or access points.

- Forwarding Node (FN): The forwarding node is the second tier that offers multi-hop routing capability to nearby SN's or other FN's. The FN has two wireless interfaces, one communicates with lower tier nodes (SN's) and the other connects to higher tier nodes (FN's and AP's).

- Access Point (AP): The access point is the highest tier in the network, and has both wireless and wired interfaces (similar to those used in conventional $802.11 \mathrm{~b}$ wireless LAN's). AP's provide multi-hop routing for packets from SN's and FN's within radio range, in addition to routing data to and from the wired Internet.

\section{Routing Protocols And Metrics}

In this work, we focus on the system performance of the hierarchical ad hoc network described in Section II. We consider two routing protocol alternatives: DSR and AODV. It is observed that in addition to choice of routing protocol, system performance will depend upon MAC, discovery and radio physical layer assumptions. Here we assume an 802.11based network in which all nodes use the standard $802.11 \mathrm{~b}$ adhoc mode MAC with specified power and range. The discovery protocol used in the system is assumed to provide an idealized hierarchical network topology, and then maintain and optimize the topology, which may change due to the node movements and varying network traffic.

Both DSR and AODV are designed for "flat" ad hoc networks. Thus, it may be necessary to make some changes in order to apply them to the proposed hierarchical ad hoc network. The main modification is for SN's, which do not offer multi-hop routing capability to other nodes. During the route discovery procedure of both DSR and AODV, SN's ignore route request messages they may receive. In addition, in DSR, when a node operating in promiscuous mode overhears a route, before it adds this route to its cache, it first makes sure that there is no other SN contained in the route. In AODV, SN's update their routing tables after they receive the route error messages, but they do not continue to broadcast or unicast the route error messages and just drop them. These modifications assure that any $\mathrm{SN}$ will not be used as an intermediate node to forward packets for other nodes.

Most of the routing protocols, including DSR and AODV, use the number of hops as the metric to make routing decisions. This metric tends to choose the route with the minimum data packet delay. In addition to delay, we may have to consider other system performance factors such as throughput and energy consumption. When calculating the routing costs, the energy consumption of nodes, the number of hops reaching the destination, the available radio bandwidth, the link latency and the network traffic load, can all be used as parameters. It may also be possible to devise integrated MAC/routing policies with metrics related to dynamically observed MAC layer parameters such as congestion or channel quality.

Since ad hoc networks have specific requirements for minimizing energy consumption at nodes and/or maximizing network lifetime, energy-aware routing has received attention in the recent few years [4]. We start by using an energyaware metric in DSR, and identify protocol and algorithmic extensions necessary for efficient operation in the hierarchical environment. Then we study the hierarchical system capacity and performance with the energy-aware metric.

\section{Methodology and Simulation Model}

Hierarchical ad hoc network system capacity and performance are evaluated for an example "sensor network" scenario using the Monarch extensions to the $n s-2$ network simulator [5]. First we use modified DSR and compare the results with those of a conventional "flat" ad hoc network in order to estimate the potential increase in system capacity with the three-tier hierarchy. Then we use modified AODV and study the hierarchical behavior with an alternative routing protocol.

\section{A. Hierarchical Ad Hoc Network Model}

In the proposed hierarchical ad hoc network, an idealized discovery protocol is used to establish and maintain the hierarchical topology. First, nodes are assumed to organize themselves into clusters. For simplicity in this study, we assume that there is only one "gateway AP" per cluster associated with an arbitrary number of FN's and SN's. When an SN wishes to communicate with any other node outside its cluster, its packets must go through the gateway AP. If an FN or $\mathrm{SN}$ is within the radio transmission range of its gateway $\mathrm{AP}$, it is connected to the gateway AP directly; otherwise, the connection is through one or more intermediate FN's. We 
assume that any FN or SN can only belong to one cluster, i.e., it only has one unique gateway AP. This assumes that the discovery protocol supports identification of gateway AP's and association of related FN's and SN's in each cluster.

We assume that we have an optimized self-organizing hierarchical topology during the simulations. In particular, we assume that the clusters are created and maintained with balanced traffic load. Meanwhile, any FN or SN can move out of its original cluster and join a new cluster due to its movement; the discovery protocol will also take care of this topology change. Under these assumptions, we can simply implement the hierarchical ad hoc network by dividing the simulated site into a certain number of clusters, with the gateway AP in the center of each cluster. Our simulation scenarios are run with approximately the same number of nodes in each cluster and the same traffic pattern in the nodes.

The SN is modeled as a simple mobile node without multihop routing capability offered to neighbors. The FN has full multi-hop routing capability. But each FN only forwards packets to other nodes within the same cluster. The AP's are assumed to be connected by high-speed wired links (such as $\sim 100$ Mbps supported by an upstream Ethernet switch). Wired network congestion is ignored in our model, and the packet error rate is set to zero for wired connections. Delays caused by the wired link are obtained by dividing the packet size by the link speed. Because packets can be forwarded between AP's over high-bandwidth wired links with minimal routing cost, the gateway AP receives all packets sent by the nodes in its cluster, no matter which AP is the eventual destination.

\section{B. Hierarchical Sensor Network Simulation Model}

The simulation study considers an example sensor network deployed over a square geographical coverage area with dimension $1000 \mathrm{~m} \times 1000 \mathrm{~m}$. We divide the coverage area into four $500 \mathrm{~m} \times 500 \mathrm{~m}$ smaller squares, each corresponding to a cluster with one gateway AP and several FN's and SN's. The gateway AP is static and located in the center of each small square. Thus, there are 4 AP's in the simulated area of coverage. The FN's and SN's are randomly placed within the clusters. In this model, we assume a uniform density of SN's and FN's, with a nominal value of 20 FN's and 100 SN's spread over the entire coverage area. The FN's move according to the random waypoint model [2] with a randomly chosen speed (uniformly distributed between $0-1 \mathrm{~m} / \mathrm{s}$ ) and pause time of zero (which means that FN's do not stop during their journey). Half of the SN's are static; the remaining half move according to the same random waypoint model as the FN's. These parameters have been chosen to an example sensor network scenario, but are by no means unique. Other sensor network scenarios will be considered in the future work.

\section{Traffic Pattern}

Sensor nodes generate traffic according to an exponential on/off model [5]. Packets are sent at a specific rate during "on" periods, and no packet is sent during "off" periods. Both "on" and "off" periods are taken from an exponential distribution.
We choose the average "on" time (burst time) to be $500 \mathrm{~ms}$, the average "off" time (idle time) to be $500 \mathrm{~ms}$, and the packet size to be 64 bytes per packet. The number of packets per second generated by SN's is varied as an input parameter in order to gradually increase the offered load to the network as a whole. All traffic in the sensor network scenario is originated at SN's, and $80 \%$ of this traffic is assumed to be bound for a server within the Internet, accessed through an AP; the remaining $20 \%$ of SN traffic is assumed to be routed to other SN's in the network, accessed via one or more FN's and AP's. Each SN can simultaneously support up to two traffic flows to different destinations. Traffic in the network is assumed to be uniform and balanced.

\section{Energy Cost Metric}

Since energy is a serious constraint in sensor networks, it may be appropriate to use energy cost as an alternative routing metric. When such a metric is used, the system will tend to favor routes with multiple SN-FN-FN-AP hops rather than a direct high-power link from SN to AP. We note that use of energy-aware routing metric implies the existence of reasonably effective power control at the $802.11 \mathrm{~b}$ link/physical level. Such power control may also be expected to improve MAC layer efficiency in the network.

\section{Performance Results}

\section{A. Performance Metrics}

Four important performance metrics are evaluated:

- Packet delivery fraction: measured as a ratio of the number of data packets delivered to their eventual destinations and the number of data packets generated by sources.

- Average end-to-end delay: includes all possible delays before data packets arrive at their destinations.

- Normalized routing load: measured as the number of routing packets transmitted per data packet delivered at destinations. Each hop is counted as one transmission for both routing and data packets sent over multiple hops.

- System throughput: measured as the total number of useful data received at traffic destinations (in bps).

\section{B. Results and Discussions}

A series of simulation experiments for the sensor network scenario were conducted using the system model and parameters outlined in the last section. The key parameters are summarized in Table I below.

TABLE I

SimULATION PARAMETERS

\begin{tabular}{|c|c|}
\hline Simulation area & $1000 \mathrm{~m} \times 1000 \mathrm{~m}$ \\
\hline Number of clusters; AP's; FN's; SN's & $4 ; 4 ; 20 ; 100$ \\
\hline Radio PHY ; Radio range & $1 \mathrm{Mbps} ; 250 \mathrm{~m}$ \\
\hline MAC & Ad-hoc $802.11 \mathrm{~b}$ \\
\hline AP-AP wired link speed & $100 \mathrm{Mbps}$ \\
\hline Number of communication pairs & 40 \\
\hline Number of packets/sec generated & $1,4,8,12,16,24,32$ \\
\hline Packet size & 64 bytes \\
\hline Fraction of SN-Internet traffic & $100 \%$ \\
\hline
\end{tabular}


1) Simulations with DSR: From Fig. 2a which shows throughput as a function of offered load from the sensors (for $40 \mathrm{SN}-\mathrm{AP}$ communicating pairs), we see that the hierarchical system begins to saturate when the packet generation rate reaches $16 \mathrm{pkts} / \mathrm{s}$; while the flat system saturates at about $4 \mathrm{pkts} / \mathrm{s}$. For the $802.11 \mathrm{~b}$ bandwidth of $1 \mathrm{Mbps}$ used in the study, system capacities are found to be $\sim 320 \mathrm{kbps}$ for the hierarchical case and $\sim 77 \mathrm{kbps}$ for the flat case, respectively.

It is observed that the system capacity increases by a factor of $\sim 4 \times$ if the proposed hierarchical architecture is adopted. Clearly, this is a significant scaling increase over the relatively low $77 \mathrm{kbps}$ obtained with the flat network. Note that this result is obtained for a specific network model with relatively high AP density. It is expected that the gain will decrease when the number of AP's decreases. So the exact factor by which the capacity increases will depend upon the spatial distributions and the relative densities of FN's and AP's. We will consider the performance with varied AP density in Section VI. The average end-to-end delay, packet delivery fraction and routing overhead curves for system scenario summaried in Table I are shown in Fig. 2b, c and d. The simulations were repeated for two other cases corresponding to 20 and 60 communication pairs and results similar to the 40-pair case were observed.

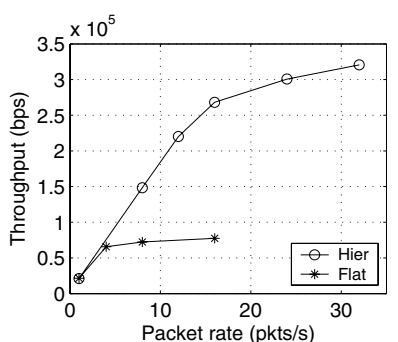

(a)

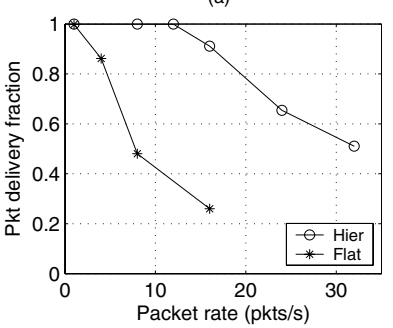

(c)

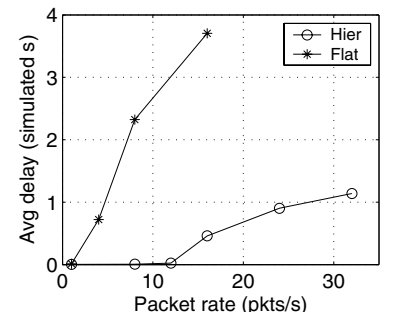

(b)

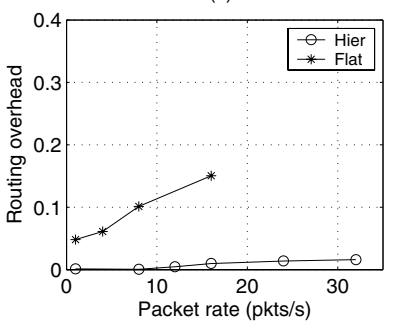

(d)
Fig. 2. Simulation results for 40 communication pairs (with DSR)

The figures show that the hierarchical network has better performance in terms of the performance metrics that we evaluate when compared with the flat ad hoc network. Each SN communicates through a few FN's and a single gateway AP, thus reducing the number of hops to reach the Internet, where most packets from sensors have their destinations $(100 \%$ in this study). In addition, SN's do not join the full distribution of routing messages, thus reducing routing overhead significantly. Of course, the capacity increase comes at the expense of increased hardware (FN's and AP's) relative to a flat network, and in that sense it is not an "apples-to-apples" comparison.

We also briefly studied the feasibility of using alternative routing metrics (such as the energy cost) within the DSR framework. Specifically, we simulated for the same model and parameters as described above, with the hop-count metric in DSR replaced by an energy metric computed from the actual power required to transmit on each radio link. In particular, the energy cost is a function of the sum of the transmission power required to reach the next hop through the path. From the results for 12 pkts/s shown in Table II, it is observed that the network with energy-cost routing metric helps reduce power consumption (the average energy cost per data packet is about $15-20 \%$ less) at the nodes at the expense of lower throughput and higher delay. This result matches our expectations, and leads us to expect that combined link metrics with a mix of both hop-count and energy-cost can be used to further tune the performance vs. energy consumption at sensor nodes.

TABLE II

SIMULATION RESULTS OF HOP-COUNT AND ENERGY-COST METIRCS

\begin{tabular}{|c|c|c|}
\hline Metric & Hop-count & Energy-cost \\
\hline Delivery fraction & 0.982 & 0.946 \\
\hline Throughput (bps) & 220084 & 212631 \\
\hline Average delay (s) & 0.0258 & 0.0454 \\
\hline
\end{tabular}

2) Simulations with AODV: Experiments were also performed with AODV using the $n s-2$ implementation from Uppsala University [7]. We used the same system model and parameters as for the DSR case described earlier. The simulation results obtained are shown in Fig. 3.

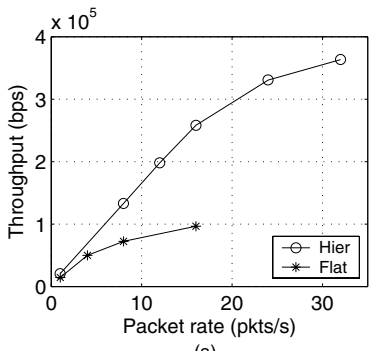

(a)

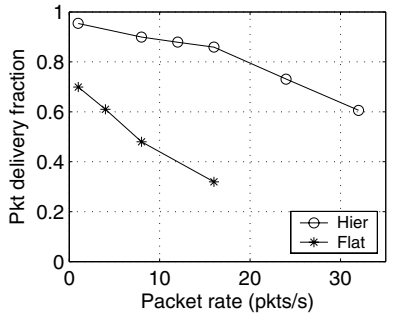

(c)
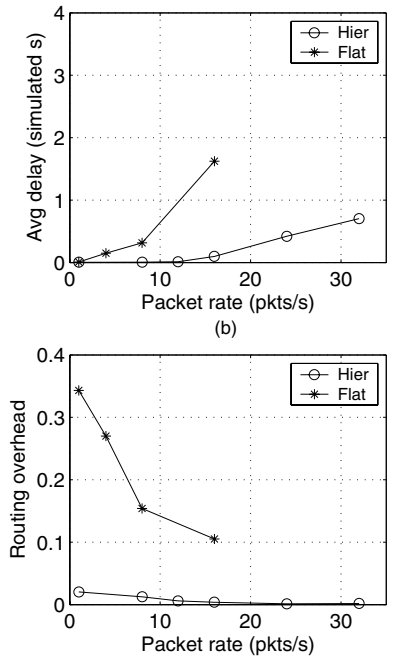

(d)
Fig. 3. Simulation results for 40 communication pairs (with AODV)

From these curves it is observed that system capacity and performance improve significantly with AODV as well as DSR. The main reason is the deployment of three-tier hierarchy and the wired integration, as discussed in the DSR case. When comparing the results, it is observed that the three-tier hierarchy provides $\sim 4 \times$ higher saturated throughput (capacity) as the flat network, and both have similar delay vs. offered load characteristics. The capacity of AODV is found to be marginally higher, and the average packet delays are also correspondingly lower. The AODV case does show a poorer 
fraction of packets delivered, possibly due to higher rates of link delivery failure. Previous simulation studies show that AODV generally has a higher routing load than DSR [6]. This is because DSR's caching is very efficient at low speed, which is our case here. AODV's routing load is dominated by route request packets. In hierarchical network, SN's do not join in the flooding of route requests, so AODV can achieve a low routing overhead comparable to DSR. At the same time, fewer routing packets decrease the chance of link delivery failure, which results in higher data packet delivery fraction in the hierarchical mode.

We conclude that the performance and capacity of the threetier network are relatively insensitive to the choice of routing protocol, and that both DSR and AODV are viable choices. AODV appears to have marginally higher capacity and lower delay than DSR, but with lower packet delivery fraction for the particular scenario and configuration parameters.

\section{Scaling Behavior of Hierarchical CAPACITy}

Our experiments have shown that the system capacity increases by a fairly large multiplier when the proposed hierarchical architecture is adopted. In addition to topology and traffic pattern, which are two important factors for the ad hoc network capacity [8], the precise capacity scaling factor depends upon the spatial distributions and the relative densities of FN's and AP's. In this part of the study we focus on this question: how to design a three-tier hierarchical ad hoc network to obtain scalable capacity while minimizing the investment in new devices.

For the three-tier hierarchy under consideration, we observe that the core ad hoc network is formed by FN's and AP's, while SN's feed traffic into nearby AP's or FN's. So the system capacity depends only on the density of traffic offered by SN's and not their exact number. As a result, the key input parameters for the system's scaling performance are the SN's offered traffic load density (define this as $G_{S}=$ $\lambda_{S} \times L_{S}$, where $\lambda_{S}$ is SN's packet arrival rate per unit area in pkts/sec/ $m^{2}$, and $L_{S}$ is the average packet length in bits/pkt), the density of FN's (denoted $\mathcal{X}_{F N}$ ) and the density of AP's (denoted $\mathcal{X}_{A P}$ ). Clearly, the numbers of FN's and AP's cannot be selected arbitrarily given that FN's and AP's must cover the entire service area to ensure that all SN's are reachable. Note also that in the three-tier network, an AP represents a significantly higher investment than an FN, so we adopt the heuristic approach of covering the entire service area with FN's and then determining the right number of AP's necessary for the network to scale in a balanced way. As shown in Fig. $4 \mathrm{a}$, we obtain the system capacity by observing the network throughput as a function of increasing $G_{S}$ while varying $\mathcal{X}_{A P}$, given a fixed value of $\mathcal{X}_{F N}$.

\section{A. Simulation Model}

We continue to use the hierarchical ad hoc network model and assumptions described in Section IV-A. For the simulation model, we consider a square simulated region, where FN's are evenly placed within the square and form a regular planar network. Note that although this regular spatial model is expected to produce optimistic results relative to random spatial model, it is considered useful for estimating the achievable capacity, as in [9]. The locations of FN's are fixed and the distances between neighboring FN's are all 200 meters. With the specified $802.11 \mathrm{~b}$ transmission range of 250 meters (AP's and SN's also use the same transmission range), this FN density provides full coverage over the simulated region. We use two simulation cases for coverage areas with dimension of $1200 \mathrm{~m} \times 1200 \mathrm{~m}$ and $800 \mathrm{~m} \times 800 \mathrm{~m}$ respectively in order to explore the sensitivity to physical assumptions. Since the density of FN's is identical for the two dimensions, there are a total of 36 FN's for the $1200 m \times 1200 m$ case and 16 FN's for the $800 m \times 800 m$ case.

The number of AP's is now varied for each of these two simulation scenarios to see how the capacity of the hierarchical network changes with the ratio of FN's to AP's. A regular planar network topology formed by 16 FN's (suppose 4 AP's here) over an $800 \mathrm{~m} \times 800 \mathrm{~m}$ field is depicted in Fig. $4 \mathrm{~b}$. The second $1200 \mathrm{~m} \times 1200 \mathrm{~m}$ scenario with $36 \mathrm{FN}$ 's (suppose 2 AP's) is shown in Fig. 4c.

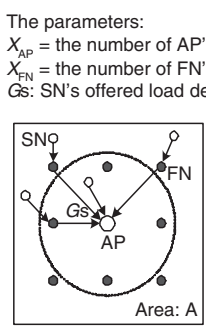

(a)

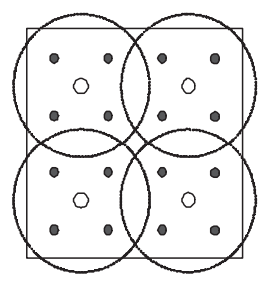

(b)

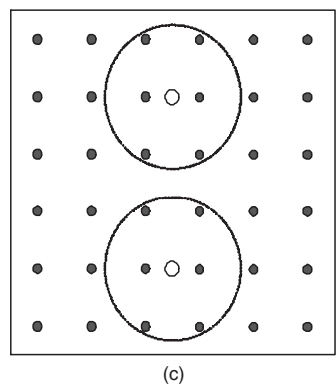

Fig. 4. Parameter definitions and the regular planar network topology

SN's are randomly placed in the network, and move according to the random waypoint model with a randomly chosen speed (uniformly distributed between $0-1 \mathrm{~m} / \mathrm{s}$ ) and pause time of zero. So all the SN's move slowly and continuously during the simulations. The density of SN's in the network is identical for all the experiments. Each cluster has the same number of SN's. Each SN generates traffic according to the exponential on/off model as we described in Section IV-C. We use the same parameters as before, and vary the number of packets per second generated by each $\mathrm{SN}$ as an input parameter in order to gradually increase the offered load density to the network. DSR is used for the hierarchical capacity study.

Table III lists the key parameters. Parameters not listed in this table such as the physical, MAC and wired link parameters, are the same as those of Table I.

TABLE III

PARAMETERS FOR CAPACITY SIMULATIONS

\begin{tabular}{|c|c|c|}
\hline Simulation area & $1200 m \times 1200 m$ & $800 m \times 800 m$ \\
\hline Number of AP's & $1,2,4,6,9,12$ & $1,2,4,6$ \\
\hline Number of FN's & 36 & 16 \\
\hline Number of SN's & 90 & 40 \\
\hline Number of pkts/s generated & \multicolumn{2}{|c|}{$1,4,8,12,16,24,32,40$} \\
\hline
\end{tabular}




\section{B. Simulation Results and Discussions}

In this hierarchical capacity study, we measure the normalized system throughput (per unit area) as a function of the normalized offered load, and thus obtain the normalized system capacity (i.e. the maximum throughput).

Simulation results for 36 FN's and $1200 m \times 1200 m$ field are shown in Fig. 5. Fig. 5a shows that the normalized system throughput increases when the number of AP's increases from 1 to 9. Observe that once the number of AP's reaches 4, the system throughput tends to increase at a slower rate. As expected, the highest capacity is obtained with 9 AP's, since 9 AP's give the full coverage over the simulated site in our model. When the number of AP's is greater than 9 , the overlapping of the coverage areas of the neighboring AP's becomes a factor, which results in interference between AP's thus the normalized capacity begins to decrease. The normalized capacity with 12 AP's is even less than that with 4 AP's for the light offered load, but it starts to outperform others (except for 9 AP's case) when the offered load increases. We also observe that the average end-to-end delay decreases when the number of AP's increases.

Fig. 5b shows the achievable normalized capacity with different numbers of AP's. The curve without delay constraints represents the capacity result we have discussed. When we bound the permissible packet delay to a specified value, we can also obtain the corresponding normalized capacity with this bounded delay. Since the delay decreases when the number of AP's increases, we can get different curves when we choose different values of bounded delay. But these curves have similar behavior and all demonstrate a saturation phenomena starting at 4 AP's.

We observe that the normalized capacity increases almost linearly before the number of AP's increases to $\sim 4$, while the curve saturates rather rapidly as the number of AP's is increased futher. Clearly, the "knee" of the capacity vs. the number of AP's curve is a good operating region for system designers because it achieves near-maximum network capacity with a modest investment in access points (which are a more costly network element than FN's due to the need for a wired interface). In this case, a system designer should aim to provision the network with $\sim 4-6$ AP's for a region that requires $\sim 36$ FN's for full coverage.

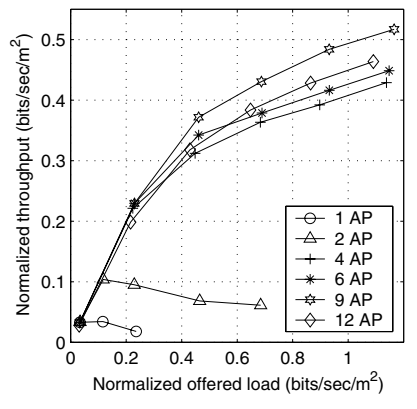

(a) Throughput vs. offered load

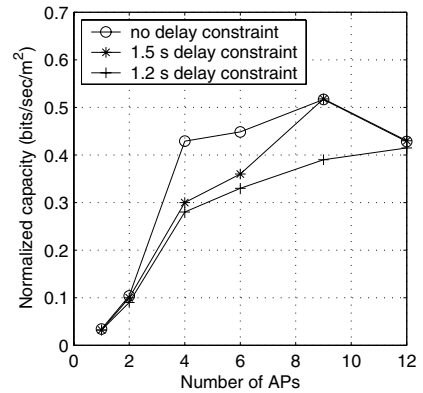

(b) Capacity vs. number of AP's
Fig. 5. Capacity simulation results $(1200 m \times 1200 m, 36$ FN's $)$

We repeated the experiments for $16 \mathrm{FN}$ 's and $800 \mathrm{~m} \times 800 \mathrm{~m}$ field and observed similar results. In this case, the knee of the capacity curve is reached with $\sim 3-4$ AP's. It is also observed that the achievable normalized capacities of these two cases are comparable, as might be expected as the simulated region grows larger. While this is still speculative, a rough squarelaw relationship between the density of FN's and AP's (i.e. $\mathcal{X}_{A P} \cong k \sqrt{\mathcal{X}_{F N}}$, where $k$ is a constant) may be inferred from these preliminary results. Such a square law ratio would also be consistent with the analytical capacity scaling result obtained by Liu and Towsley in [10].

\section{CONCLUSIONS AND FUTURE WORK}

We have compared the performance of traditional flat ad hoc networks with a novel three-tier hierarchical approach. Significant improvements in performance and system capacity have been demonstrated for an example sensor network scenario with $802.11 \mathrm{~b}$ radios. Both DSR and AODV protocols seem to work well for the hierarchical network, with AODV performing marginally better in terms of capacity and packet delay in the specific examples considered. The simulations also show that modified routing metrics can be used to trade off throughput and delay against energy consumption.

We have also studied how the three-tier hierarchical network's capacity scales as a function of the relative densities of forwarding nodes and access points. These results indicate that it is possible to scale network capacity quite well with a mix of several (lower-cost) radio forwarding nodes and just a few wired access points. In future work, we plan to validate these capacity scaling observations with a more general analytical model. In addition, we intend to further investigate routing protocols for the three-tier hierarchical ad-hoc network with a focus on integrated MAC/routing issues not considered here. Finally, results from ongoing experimental prototyping of the three-tier hierarchical ad-hoc network concept will also be reported in future work.

\section{REFERENCES}

[1] P. Gupta and P. R. Kumar, "The capacity of wireless networks," IEEE Trans. on Information Theory, vol. 46, March 2000, pp. 388-404.

[2] D. B. Johnson and D. A. Maltz, "Dynamic source routing in ad hoc wireless networks," Mobile Computing, T. Imielinski and H. Korth, Eds., Kluwer Publishers, 1996, ch. 5, pp. 153-181.

[3] C. E. Perkins and E. M. Royer, "Ad hoc on-demand distance vector routing," Proc. IEEE Workshop on Mobile Computing Systems and Applications, 1999, pp. 90-100.

[4] S. Singh, M. Woo, and C. S. Raghavendra, "Power-aware routing in mobile ad hoc networks," MOBICOM, 1998, pp. 181-190.

[5] K. Fall and K. Varadhan, Eds., "The ns Manual (2002)," The VINT Project, UC Berkeley, LBL, USC/ISI, and Xerox PARC. [Online]. Available WWW: http://www.isi.edu/nsnam/ns/doc/ns_doc.pdf

[6] C. E. Perkins, E. M. Royer, S. R. Das, and M. K. Marina, "Performance comparison of two on-demand routing protocols for ad hoc netwroks," IEEE Personal Communications, Feb. 2001, pp. 16-28.

[7] AODV-UU: The AODV routing protocol implementation by Uppsala University, Available WWW: http://user.it.uu.se/ henrikl/aodv/

[8] J. Li, C. Blake, D. S. J. De Couto, H. I. Lee, and R. Morris, "Capacity of ad hoc wireless networks," Proc. ACM SIGMOBILE, 2001, pp. 61-69.

[9] L.-L. Xie and P. R. Kumar, "A network information theory for wireless communications: scaling laws and optimal operation," To appear in IEEE Trans. Inform. Theory, April 2002, Revised Nov. 2003.

[10] B. Liu, Z. Liu, and D. Towsley, "On the capacity of hybrid wireless networks," IEEE INFOCOM 2003, March 2003. 